\title{
Resiliencia en trabajadores y en residentes de un centro de personas mayores institucionalizadas
}

\author{
Inmaculada Méndez ${ }^{1}$, Julia García-Sevilla ${ }^{1}$, Juan P. Martínez², Ma Ángeles \\ Boti $^{1}$, Ana B. Cánovas ${ }^{1}$ y Yolanda Clemente ${ }^{1}$ \\ ${ }^{1}$ Universidad de Murcia (España); ${ }^{2}$ Equipo de Orientación Educativa y \\ Psicopedagógica Murcia 2 (España)
}

La resiliencia supone un constructo dinámico y multidimensional que alude a la capacidad que tienen las personas para afrontar o recuperarse con éxito de las situaciones adversas. El objetivo del presente estudio ha sido analizar la capacidad de resiliencia en trabajadores y en residentes de un centro de personas mayores institucionalizadas en función de las características sociodemográficas (sexo, edad y estado civil) tras la situación adversa por el terremoto ocurrido el Lorca en mayo del 2011. Se ha administrado un cuestionario sociodemográfico desarrollado ad hoc junto a la adaptación de la Escala de Resiliencia de Connor-Davidson (CD-RISC) en los sujetos del estudio. Los participantes fueron 20 trabajadores y 34 personas mayores de un centro de personas mayores institucionalizadas de la Región de Murcia (Lorca). La edad media de las personas mayores institucionalizadas fue de 81 años con una desviación típica de 7.68 y la edad media de los cuidadores profesionales fue de 37.85 años y la desviación típica de 9.60. El estudio permite determinar la capacidad de resiliencia atendiendo a características sociodemográficas así como la existencia de diferencias de medias significativas entre la capacidad de tenacidadautoeficacia entre los trabajadores y los residentes del centro de personas mayores lo que permitirá establecer actividades de prevención y/o de intervención.

Palabras clave: Resiliencia, tenacidad y autoeficacia, control personal, personas mayores, competencia social, cuidadores profesionales.

Resilience in workers and residents of a Centre for the Elderly institutionalized. Resilience is a dynamic and multidimensional construct that refers to the ability of people to cope with or recover successfully from adverse situations. The aim of this study was to analyze resilience in workers and residents of a Centre for the Elderly institutionalized according to sociodemographic characteristics (sex, age and marital status) after the adverse situation caused by an earthquake in Lorca in May 2011. A sociodemographic questionnaire developed $a d$ hoc and the adaptation of the Resilience Scale Connor-Davidson (CD-RISC) were administered. Participants were 20 workers and 34 seniors in a Centre for the Elderly institutionalized in the Region of Murcia (Lorca). The average age of the institutionalized seniors was 81 years with a standard deviation of 7.68 and the average age of professional caregivers was 37.85 years with a standard deviation of 9.60. The study allows to determine resilience capacity in response to sociodemographic characteristics and the existence of significant mean differences between the ability of tenacity-efficacy among workers and residents of the Centre for the Elderly. This will allow to design actions to prevent and/or intervene promptly.

Keywords: Resilience, tenacity and self-efficacy, personal control, seniors, social competence, professional caregivers.

Correspondencia: Inmaculada Méndez. Facultad de Psicología. Departamento de Psicología Evolutiva y de la Educación. Universidad de Murcia. Campus de Espinardo. C.P.: 30100. Murcia (España). E-mail: inmamendez@um.es 
La resiliencia supone un constructo dinámico que involucra la interacción entre los procesos de riesgo y de protección tanto a nivel interno como externo que pone en marcha el individuo para combatir los efectos adversos a los que se enfrenta para superar el riesgo a lo largo del ciclo vital (González-Arratia y Valdez, 2013). Los estudios realizados son contradictorios en lo que respecta a las características sociodemográficas tales como la edad y el sexo así como en los instrumentos de evaluación (Cárdenas-Jiménez y López-Díaz, 2011). Parece ser que los factores subyacentes a la resiliencia se conservan e incluso aumentan conforme las personas mayores avanzan en la edad (Nygren et al., 2005). Con respecto al sexo, existen estudios que determinan que las mujeres puntuaron más alto en el factor protector externo, es decir, requieren de mayor apoyo externo en diferentes edades, frente a los hombres que puntuaron más alto en el factor protector interno (González-Arratia y Valdez, 2013).

Entre las etapas del ciclo vital, si nos centramos desde la juventud plena hasta la vejez, se han de destacar las situaciones a las que enfrentarse en cada una de ellas. Por un lado, las personas durante la juventud plena y la madurez han de enfrentarse al mundo laboral, cultivar vínculos afectivos, cuidar a la nueva generación, etc. Por otro lado, las personas mayores tienen que enfrentarse a un período del ciclo vital que supone afrontar situaciones tales como: muerte de seres queridos, jubilación, disminución de la autonomía, disminución de la situación económica (González-Arratia y Valdez, 2013), aumento de la dependencia (Gázquez, Pérez-Fuentes, Mercader y Molero, 2011), etc. Además, se ha de considerar el hecho de que la sensación de estrés aumenta cuando las personas mayores han de enfrentarse a contextos que les exigen adaptarse a los mismos, como por ejemplo, realizar la transición desde el hogar hasta una institución (Molina, Meléndez y Navarro, 2008).

Atendiendo al cuidado de las personas mayores, Fernández-Lansac, Crespo, Cáceres y Rodríguez-Poyo (2012) resaltan que los cuidadores no profesionales se enfrentan exitosamente a los estresores derivados del cuidado, obteniendo beneficios de su experiencia, mostrándose resilientes, es decir, se asocia una mayor resiliencia con un mejor estado emocional y físico.

En el ámbito de un centro institucionalizado para personas mayores, Méndez, Martínez, Secanilla, Benavente y García-Sevilla (2013) destacan que la calidad de vida de las personas mayores del centro de Lorca fue sensiblemente inferior con respecto a los residentes de Barcelona. Esas diferencias pudieron deberse a la situación de estrés vivida tras el terremoto de Lorca del 11 de mayo del 2011 ya que las personas mayores tuvieron que vivir cambios drásticos en sus vidas, en sus rutinas y hábitos tras el seísmo al ser realojados hasta la finalización de las obras. Del mismo modo, Martínez, Méndez, Secanilla, Benavente y García-Sevilla (2014) resaltan que los cuidadores profesionales de dicho centro tuvieron que hacer frente a la situación adversa lo que supuso una mayor entrega y dedicación en aras de luchar, así como una mayor realización profesional. 
Tras lo expuesto, el objetivo del presente estudio ha sido comparar las dimensiones de resiliencia en cuidadores profesionales y en personas mayores que residen en un centro de personas mayores institucionalizadas en función de las características sociodemográficas (sexo, edad y estado civil) tras la situación adversa por el terremoto ocurrido el Lorca en mayo del 2011.

\section{MÉTODO}

\section{Sujetos}

Los sujetos del estudio fueron 54 participantes entre trabajadores y personas mayores de un centro de personas mayores institucionalizadas de la Región de Murcia (Lorca). Por un lado, en lo que respecta a las personas mayores institucionalizadas, de 79 residentes participaron en el estudio un total de 34 sujetos con una edad comprendida entre los 66 años y los 95 años siendo la edad media de 81 años y una desviación típica de 7.68 (véase figura 1). Por otro lado, en cuanto a los trabajadores de la residencia hubo un total de 20 sujetos, de los 44 trabajadores de la residencia, con una edad comprendida entre los 21 y 61 años siendo la edad media de 37.85 años y la desviación típica de 9.60. En cuanto al sexo, el $22.2 \%$ eran hombres (siendo el $20.4 \%$ de residentes) y el $77.8 \%$ mujeres (siendo $42.6 \%$ de residentes). Según el estado civil de los sujetos se obtuvo que el $29.6 \%$ estaban casados (siendo el $7.4 \%$ residentes); el $5.6 \%$ separados o divorciados (siendo el $3.7 \%$ residentes), el $42.6 \%$ viudos (siendo todos residentes) y el $22.2 \%$ separado o divorciado (siendo el $9.3 \%$ residentes). Todos los sujetos eran de procedencia española a excepción de un residente que era de procedencia extranjera.

Con relación a las personas mayores, se utilizó un muestreo por conveniencia atendiendo a unos criterios de inclusión o exclusión en el estudio. Para ello, el criterio de inclusión fue en primer lugar, estar residiendo en la institución bajo estudio y en segundo lugar no poseer deterioro cognitivo moderado asegurando que los sujetos fuesen capaces de realizar el instrumento de evaluación. Por lo tanto, el criterio de exclusión fue presentar un nivel de deterioro cognitivo de demencia grave o muy severo según la Global Deterioration Scale -GDS- (Reisberg, Ferris, León y Crook, 1982).

De este modo, atendiendo a la Global Deterioration Scale de Reisberg et al. (1982) las personas mayores se distribuyeron del siguiente modo: el $47.1 \%$ en GDS 1 (sin deterioro cognitivo); el $8.8 \%$ en GDS 2 (deterioro cognitivo muy leve); el $20.6 \%$ en GDS 3 (deterioro cognitivo leve); el $11.8 \%$ en GDS 4 (deterioro cognitivo moderado) y el $11.8 \%$ en GDS 5 (deterioro cognitivo moderadamente grave).

En lo que respecta a los cuidadores profesionales, la distribución por puesto de trabajo fue la siguiente: $5 \%$ personal de dirección; $45 \%$ auxiliar de enfermería o enfermería; $25 \%$ personal de trabajo social, fisioterapeuta y psicólogo; el 10\% personal de lavandería, cocina, limpieza o mantenimiento; el $10 \%$ gerocultor o velador y $5 \%$ de 
otro tipo. Los turnos de los trabajadores eran los siguientes: $55 \%$ turno de mañana; $15 \%$ turno de tarde; $5 \%$ turno de noche; $15 \%$ jornada completa y $10 \%$ turnos rotativos. El tipo de contrato de los trabajadores se distribuía del siguiente modo: $55 \%$ personal fijo; $15 \%$ personal temporal; $20 \%$ de prácticas y $10 \%$ de otro tipo. Según el nivel de estudios de los trabajadores se obtuvo que el $40 \%$ poseían el graduado escolar, bachillerato elemental o formación profesional de primer grado; el 10\% bachillerato superior o formación profesional de segundo grado y el $50 \%$ estudios universitarios.

Figura 1. Histograma de edad para el total de los sujetos

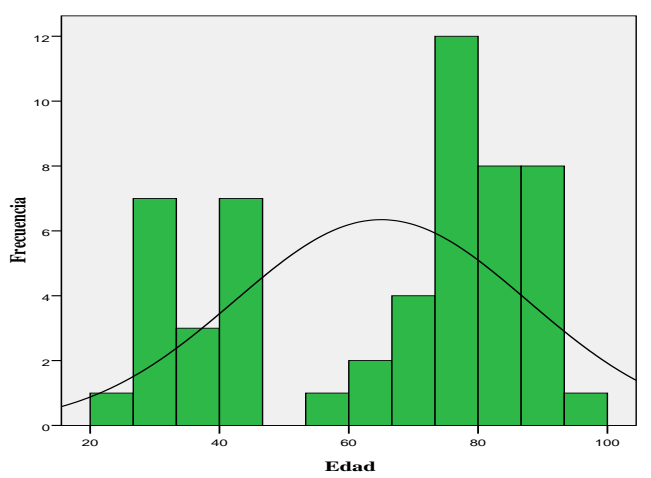

Media $=65,02$ Desviación típica $\mathrm{N}=54$

\section{Instrumentos}

Con la finalidad de recabar datos sobre características sociodemográficas se estimó oportuno obtener información a través de un cuestionario elaborado ad hoc sobre las siguientes variables: sexo, edad y estado civil. En el caso de los cuidadores profesionales se recabo información sobre el puesto de trabajo, turnos y nivel de estudios.

Para valorar la resiliencia se ha utilizado la Escala de Resiliencia CD-RISC (Connor y Davidson, 2003) traducida al español por Bobes et al. (2001) citado en Serrano-Parra et al. (2012) y validada en personas mayores de 60 años por Serrano-Parra et al. (2012). La escala consta de 17 ítems tipo Likert (0 nunca, 1 casi nunca, 2 a veces, 3 casi siempre y 4 siempre). El instrumento evalúa las siguientes dimensiones Tenacidad-Autoeficacia; Control personal y Competencia social.

El instrumento ha mostrado buenas propiedades psicométricas siendo el alfa de Cronbach de .89 en el estudio de Connor and Davidson (2003); .79 para las dimensiones Tenacidad-Autoeficacia y Control personal y de .56 para Competencia social en la validación de Serrano-Parra et al. (2012) y para los sujetos del estudio que presentamos el alfa de Cronbach fue de .78. 


\section{Procedimiento}

Tras la selección del centro de personas mayores bajo estudio, en primer lugar se llevó a cabo una entrevista con la persona que dirige el centro de Lorca (Murcia) para detallar los objetivos del estudio así como los instrumentos de evaluación.

La selección de las personas mayores participantes en el estudio, se llevó a cabo siguiendo un muestreo por conveniencia dependiendo del cumplimiento de los criterios de inclusión mencionados. La participación de los participantes en el estudio fue voluntaria, salvaguardando la confidencialidad y anonimato. La recogida de datos se llevó a cabo durante los meses de marzo y abril del año 2014 por parte de los autores del estudio. Se ha utilizado un enfoque no experimental. Para ello se han aplicado dos instrumentos de medida en personas mayores institucionalizadas y en cuidadores profesionales de personas mayores en un centro de Lorca (Murcia).

Para el tratamiento estadístico de los datos se utilizó el programa SPSS (v. 19). El análisis descriptivo de los datos se llevó a cabo utilizando el estudio de frecuencias en porcentajes, media y desviación típica. En el caso de variables de tipo cuantitativo y la variable de agrupamiento categórica con dos grupos, se utilizó el análisis de diferencias de medias utilizando la prueba $t$ de Student para muestras independientes. Para variables con más de dos niveles de agrupación con respecto a una variable cuantitativa se seleccionó la prueba Anova de un factor para la comparación de medias. Para ambas pruebas se comprobó previamente el supuesto de homogeneidad de varianzas de Levene. Al tratarse de variables cuantitativas se utilizaron correlaciones bivariadas a través del coeficiente de correlación de Pearson.

\section{RESULTADOS}

\section{Características sociodemográficas y resiliencia}

Atendiendo al total de participantes, en cuanto al sexo no se obtuvieron diferencias de medias significativas en la prueba $t$ de Student y los factores de resiliencia. Si bien es cierto que entre las personas mayores aparecieron diferencias de medias significativas, asumiendo varianzas iguales, entre el sexo y el factor tenacidad autoeficacia siendo $t_{(32)}=2.492, p=.018$. La media de los hombres fue superior a la media de las mujeres (véase tabla 1 ).

Se ha encontrado una correlación de Pearson significativa negativa de magnitud media entre el factor de tenacidad-autoeficacia y la edad del total de participantes, trabajadores y residentes $(r=-.400 ; p=.003 ; n=54)$. En la figura 2 , se muestra la tendencia negativa entre ambas variables. 
Tabla 1. Medias y desviaciones típicas según sexo y estado civil en las dimensiones de resiliencia en trabajadores y residentes

\begin{tabular}{lcccccc}
\hline Niveles de las & \multicolumn{2}{c}{ TENACIDAD Y AUTOEFICACIA } & \multicolumn{2}{c}{ CONTROL PERSONAL } & \multicolumn{2}{c}{ COMPETENCIA SOCIAL } \\
\cline { 2 - 7 } $\begin{array}{l}\text { variables sexo } \\
\text { y estado civil }\end{array}$ & RESI. & TRAB. & RESI. & TRAB. & RESI. & TRAB. \\
\hline Hombre & $23.73(7.03)$ & 31 & $21.18(7.25)$ & 22 & $6.45(1.91)$ & 4 \\
Mujer & $17.91(6.04)$ & $24.68(4.79)$ & $19.65(5.79)$ & $18.58(3.17)$ & $5.61(2.27)$ & $5.32(2.31)$ \\
\hline Casado & $24.75(3.86)$ & $24.08(4.98)$ & $20.25(4.50)$ & $18.64(2.77)$ & $6(.82)$ & $4.75(2.00)$ \\
Separado/Div. & $27.50(6.36)$ & 28 & $26(1.41)$ & 15 & $7.50(.71)$ & 2 \\
Viudo & $19.13(6.26)$ & - & - & - & $5.57(2.45)$ & - \\
Soltero & $15.80(8.56)$ & $26.14(4.98)$ & $20(6.26)$ & $19.43(3.18)$ & $6.60(1.67)$ & $5.25(2.27)$ \\
\hline
\end{tabular}

Figura 2. Gráfico de dispersión para la edad y la tenacidad-autoeficacia

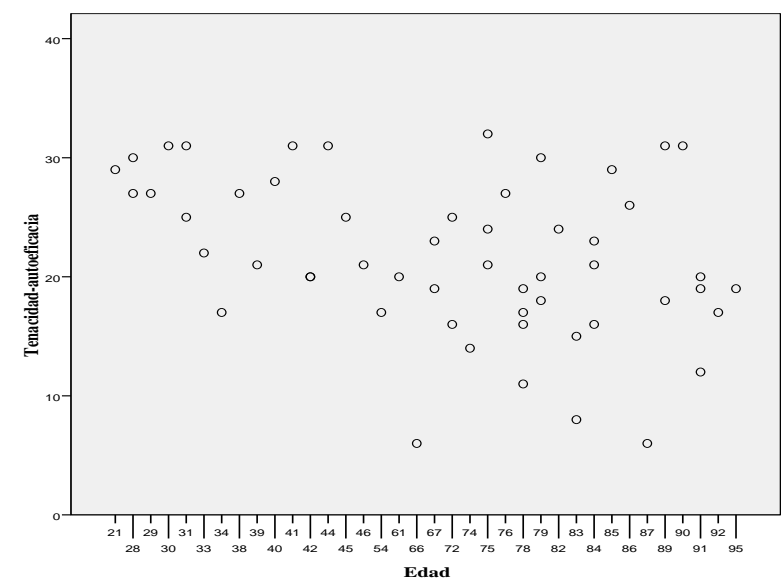

No se encontraron diferencias significativas en la prueba Anova de un factor entre el estado civil de los residentes ni en el estado civil de los trabajadores y los factores de resiliencia. Véase en la tabla 1 las medias y desviaciones típicas.

\section{Dimensiones de resiliencia}

Se ha hallado una correlación significativa positiva de magnitud media entre el factor tenacidad -autoeficacia y el factor control personal ( $r=.374 ; p=.005 ; n=54)$. Esto supone que conforme aumenta el grado de tenacidad-autoeficacia, aumenta el nivel de control personal. Del mismo modo, se encontró una correlación de Pearson significativa positiva de magnitud media entre el factor de tenacidad-autoeficacia y el factor de competencia social $(r=.341 ; p=.012 ; n=54)$. Asimismo, existía una correlación de Pearson significativa positiva de magnitud moderada entre el factor de control personal y el factor competencia social $(r=.532 ; p=.000 ; n=54)$. 


\section{Resiliencia en cuidadores profesionales y en personas mayores}

Según la prueba $t$ de Student, asumiendo varianzas iguales, se obtuvieron diferencias de medias significativas en el factor tenacidad-autoeficacia al comparar los trabajadores con las personas mayores $t_{(52)}=-2.981, p=.004$. La media de los trabajadores $(M=25 ; D T=4.87)$ fue superior a la media de las personas mayores $(M=19.79$; $D T=6.848)$. Sin embargo, no existían diferencias de medias significativas entre los factores de control personal y competencia social al comparar los trabajadores con las personas mayores.

\section{DISCUSIÓN Y CONCLUSIONES}

En lo que respecta a las características sociodemográficas, no se obtuvieron diferencias significativas entre el sexo en el conjunto de los participantes (trabajadores y personas mayores) y los factores de resiliencia. Sin embargo, entre los residentes aparecieron diferencias significativas puesto que la media de los varones fue superior a la media de las mujeres en el factor de tenacidad-autoeficacia. Estos resultados están en concordancia con lo aportado por González-Arratia y Valdez (2013) lo que denotaría que los hombres puntúan más alto en el factor protector interno.

Del mismo modo, los resultados revelan que la resiliencia se da a lo largo del ciclo vital puesto que se mantiene en las personas mayores. Nuestros resultados determinan que el aumento de edad en las personas mayores supone una disminución en el factor de tenacidad-autoeficacia. Estos resultados están en la línea con los datos que apuntan algunos estudios al afirmar que los factores subyacentes a la resiliencia se mantienen conforme las personas mayores avanzan en la edad (Nygren et al., 2005), aunque en nuestros datos se produce una disminución.

El estado civil no ha mostrado diferencias significativas en los factores de resiliencia lo que supone que los valores de resiliencia son independientes del estado civil tanto de los trabajadores como de las personas mayores.

$\mathrm{Al}$ analizar los factores de resiliencia y las correlaciones entre los mismos, hemos podido determinar que el factor tenacidad-autoeficacia aumenta conforme aumenta el factor de control personal, es decir, el aumento de la percepción de autocontrol cognitivo y emocional. El factor de tenacidad-autoeficacia también aumenta conforme aumenta el factor de competencia social, lo que supone un aumento de las redes de apoyo lo que permite optimizar los recursos y poder compartir y gestionar la experiencia acumulada ante la adversidad. Finalmente, el aumento en el factor de control personal correlaciona positivamente con el factor competencia social. A este respecto, los datos estarían en la línea de lo aportado por Calderón et al. (2004) al considerar la resiliencia como un rasgo disposicional siendo el afrontamiento adaptativo promovido por el contexto social. 
Al comparar los cuidadores profesionales con los residentes se obtuvo que la media de los trabajadores fue superior en el factor tenacidad-autoeficacia. Esto supone que los trabajadores poseen una mayor capacidad para enfrentarse a las situaciones de estrés en su labor con las personas mayores con o sin deterioro cognitivo. No siendo así con los factores de control personal y competencia social. Estos datos están en la línea de lo aportado por Fernández-Lansac et al. (2012) al resaltar que los cuidadores se enfrentan de forma exitosa a los estresores derivados del cuidado de las personas mayores, lo que beneficia su experiencia, mostrándose de este modo resilientes.

Para concluir, cabría resaltar que la resiliencia ha mostrado estar presente tanto en los cuidadores profesionales como en las personas mayores lo que permite enfrentarse a situaciones adversas como por ejemplo, el terremoto de Lorca.

Finalmente, los datos del estudio deben servir para llevar a cabo acciones preventivas o de intervención tanto en los cuidadores profesionales como en las personas mayores del centro institucionalizado de Lorca. Las acciones han de ir encaminadas al aprendizaje de la situación vivida promoviendo la preparación futura que permita anticiparse (Villalba, 2003) y por lo tanto contribuyendo en la construcción de respuestas resilientes (Saavedra, Arévalo, Gajardo, Riveros y Toledo, 2012). Del mismo modo, se han de promover variables propias del cuidador tales como el estado emocional y físico de los mismos, puesto que se asocia a una mayor resiliencia (Fernández-Lansac et al., 2012). Asimismo, se ha de hacer hincapié en la importancia que tiene fortalecer aspectos psicológicos relacionados con la resiliencia para fomentar la construcción, mantenimiento así como la restauración de la salud y calidad de vida en las personas mayores (Cárdenas-Jiménez y López-Díaz, 2011).

Según García-Renedo, Mateu, Flores y Gil (2013) se ha de fomentar el cambio de perspectiva en las investigaciones sobre desastres para centrarse en un enfoque de las fortalezas humanas, es decir, conocer las características o procesos que los sujetos poseen para adaptarse a una situación novedosa tras una situación traumática.

Como prospectivas, sería adecuado aumentar el tamaño muestral del estudio incluso teniendo en cuenta el papel de otros agentes implicados como por ejemplo, los familiares o conocidos de las personas mayores. Sería adecuado tener en cuenta otras variables como por ejemplo, el nivel de deterioro cognitivo, depresión, etc.

\section{REFERENCIAS}

Calderón, A., Espinosa, A., y Techio, E.A. (2004). Resiliencia, afrontamiento, bienestar psicológico y clima socio-emocional después de los atentados del 11 de Marzo. Ansiedad y estrés, 10(2-3), 265-276.

Cárdenas-Jiménez, A. y López-Díaz, A.L. (2011). Resiliencia en la vejez. Revista salud pública, 13 (3), 528-540. Recuperado de: http://www.revistas.unal.edu.co/index.php/revsaludpublica 
Connor, K.M. y Davidson, J.R.T. (2003). Development of a new resilience scale: The ConnorDavidson resilience scale (CD-RISC). Depression and Anxiety, 18(2), 76-82.

Fernández-Lansac, V., Crespo, M., Cáceres, R., y Rodríguez-Poyo, M. (2012). Resiliencia en cuidadores de personas con demencia: estudio preliminar. Revista Española de Geriatría y Gerontología, 47(3), 102-109.

García-Renedo, M., Mateu, R., Flores, R., y Gil, J.M. (2013). La resiliencia y las víctimas de desastres. Cuadernos de crisis y emergencias, 12(1), 37-48. Recuperado de: http://www.cuadernosdecrisis.com/numeros.php

Gázquez, J.J., Pérez-Fuentes, M.C., Mercader, I. y Molero, M.M. (2011). Prevalencia de la dependencia funcional en personas mayores. Anales de Psicología, 27(3), 871-876. Recuperado de: http://revistas.um.es/analesps/index

González-Arratia, N.I. \& Valdez, J.L. (2013). Resiliencia: Diferencias por Edad en Hombres y Mujeres Mexicanos. Acta de Investigación Psicológica, 3(1), 941-955. Recuperado de: http://www.psicologia.unam.mx/pagina/es/155/acta-de-investigacion-psicologica

Martínez, J.P., Méndez, I., Secanilla, E., Benavente, A., y García-Sevilla, J. (2014). Burnout en cuidadores profesionales y calidad de vida en residentes de centros institucionalizados. European Journal of Investigation in Health, Psychology and Education, 4(1), 41-53. Recuperado de: http://www.ejihpe.es/index.php/journal/index

Méndez, I., Martínez, J.P., Secanilla, E., Benavente, A. y García-Sevilla, J. (2013). Autoconcepto y calidad de vida de personas mayores en centros residenciales. En J.J. Gázquez Linares, M.C. Pérez Fuentes, M.M. Molero e I. Mercader (Comps.), Calidad de vida, cuidadores e intervención para la mejora de la salud en el envejecimiento (pp.71-76.) Almería: Asociación Universitaria de Educación y Psicología.

Molina, C., Meléndez, J.C., y Navarro, E. (2008). Bienestar y calidad de vida en ancianos institucionalizados y no institucionalizados. Anales de Psicología, 24(2) 312-319. Recuperado de: http://www.um.es/analesps/

Nygren, B., Jonsén, A., Gustafson, Y., Norberg, A. y Lundman, B. (2005). Resilience, sense of coherence, purpose in life and self-transcendence in relation to perceived physical and mental health among the oldest old. Aging \& Mental Health, 9(4), 354-362.

Saavedra, E., Arévalo, F., Gajardo, L., Riveros, L., y Toledo, C. (2012). Autopercepción de resiliencia en familias afectadas por el terremoto de la Región Del Maule, Chile. Revista Eleuthera, 6, 82-100.

Serrano-Parra, M.D., Garrido-Abejar, M., Notario-Pacheco, B., Bartolomé-Gutierrez, R., Solera-Martínez, M., y Martínez-Vizcaíno, V. (2012). Validez de la escala de Resiliencia de Connor-Davidson (CD-RISC) en una población de mayores entre 60 y 75 años. International Journal of Psychological Research, 5(2), 49-57. Recuperado de: http://mvint.usbmed.edu.co:8002/ojs/index.php/web/index

Reisberg, B., Ferris, S.H., León de, M.J., y Crook T. (1982). The global deterioration scale for assessment of primary degenerative dementia. American Journal of Psychiatry, 139, 1136-1139.

Villalba, C. (2003). El concepto de resiliencia individual y familiar. Aplicaciones en la intervención social. Intervención Psicosocial, 12(39), 283-299. Recuperado de: http://www.psychosocial-intervention.org/\&lang=en\&numero $=\&$ articulo=\&secc $=$ all

Recibido: 27 de abril de 2014 Recepción Modificaciones: 26 de mayo de 2014 Aceptado: 16 de junio de 2014 